\title{
Reparación Ósea Utilizando Sulfato de Calcio en Defectos Óseos de Tibia de Conejos
}

\author{
Bone Repair Using Calcium Sulfate in Bone Defects of Rabbit Tibiae \\ Marila de Oliveira*; Sergio Olate ${ }^{* * * * * *}$; Leandro Pozzer*; Bélgica Vásquez ${ }^{* * * * *}$; \\ Mario Cantín** \& José Ricardo de Albergaría-Barbosa*
}

\begin{abstract}
DE OLIVEIRA, M.; OLATE, S.; POZZER, L.; VÁSQUEZ, B.; CANTIN, M. \& ALBERGARIA-BARBOSA, J. R. Reparación ósea utilizando sulfato de calcio en defectos óseos de tibia de conejos. Int. J. Morphol., 32(4):1472-1476, 2014.

RESUMEN: La regeneración ósea en reconstrucción del proceso alveolar junto al uso de implantes oseointegrables son ampliamente utilizados, existiendo una amplia variabilidad de productos. El objetivo de esta investigación es estudiar la reparación ósea asociada a un sustituto óseo a base de sulfato de calcio cubierto con membrana biológica en defectos creados en tibia de conejos. Se seleccionaron 12 conejos de entre 3 y 6 meses para ser intervenidos; de acuerdo a protocolos de anestesia ya conocidos se realizó la técnica con acceso a ambas tibias donde se realizaron 2 defectos de 2,6 $\mathrm{mm}$ de diámetro; se formaron cuatro grupos siendo el grupo I de relleno con coágulo sanguíneo, grupo II relleno con coagulo cubierto con membrana de colágeno, grupo III relleno con sulfato de calcio y grupo IV relleno con sulfato de calcio cubierto con membrana biológica. Se realizó la eutanasia a los 21 y 42 días y posteriormente se procedió a procesar y analizar láminas histológicas con tinción de hematoxilina y eosina. Se realizó la histomorfometría comparativa analizando los datos con prueba ANOVA y prueba de Turkey considerando un valor de p $<0,05$ para significancia estadística. Los resultados demostraron que todos los defectos mostraron patrones regulares de reparación ósea; los grupos rellenados con coágulo mostraron formación ósea estadísticamente menor que el presentado por los defectos rellenos con el sulfato de calcio, aunque estos últimos presentaron mayores indicadores de presencia de proceso inflamatorio; el grupo IV fue el que presentó los mejores niveles de reparación ósea a $\operatorname{los} 21$ y 42 días. Se concluye que la reparación ósea en defectos menores es viable de ser realizada con este biomaterial y que la cobertura con membrana de colágeno puede ser útil en la regeneración ósea guiada.
\end{abstract}

PALABRAS CLAVE: Biomaterial; Injerto óseo; Relleno óseo.

\section{INTRODUCCIÓN}

El sulfato de calcio es uno de los sustitutos óseos de tipo aloplásticos caracterizados por sus propiedades osteoconductoras (Choi et al., 2011); se han posicionado dentro del mercado mundial debido a que sus características químicas, similares a las encontradas en el cuerpo humano y a que no poseen procesamiento animal dentro de su composición (Laventis et al., 2014).

En defectos menores, asociados o no a implantes dentales, se han aplicado diferentes modelos terapéuticos donde uno de ellos lo vincula junto al uso de membranas biológicas (Lopez et al., 2013); clínicamente, se han presentado algunos estudios que respaldan estos sistemas terapéuticos, mientras que otros no han reportado la misma eficiencia. Variaciones como el tipo de relleno, calidad del huésped, tipo de defecto y tipo de membrana han sido indicados como elementos importantes en el éxito del tratamiento (Olate et al., 2013; Yang et al., 2014).

El uso de membrana ha sido empleado en otros estudios experimentales confirmando que en el caso de fenestraciones, el uso de sistemas absorbibles o no absorbibles son igual de eficientes permitiendo la regeneración ósea en defectos sin diferencias en los resultados (López et al.). Por otra parte, injertos autógenos son considerados aun el "patrón de oro" en la regeneración ósea, mientras que rellenos heterógenos y homógenos se utilizan en modelos clínicos y experimentales confirmando su eficiencia en algunos defectos menores (Olate et al.).

\footnotetext{
* Divisón de Cirugía Oral y Maxilofacial, Universidad Estadual de Campinas, Piracicaba, Brasil.

** División de Cirugía Oral y Maxilofacial \& CEMYQ, Universidad de La Frontera, Temuco, Chile.

**** Centro de Investigación en Ciencias Biomédicas, Universidad Autónoma de Chile, Temuco, Chile.

***** Universidad de Tarapacá, Arica, Chile.
} 
El objetivo de esta investigación es analizar la reparación ósea en defectos menores tratados con relleno sintético de sulfato de calcio cubierto con una membrana de colágeno en defectos creados en tibia de conejos.

\section{MATERIAL Y MÉTODO}

En este estudio experimental fueron utilizados 12 conejos albinos de raza New Zealand, hembras con edad de entre 3 y 6 meses. Los animales fueron divididos en 3 grupos de acuerdo al protocolo quirúrgico, manteniéndose inicialmente con alimentación sólida y agua ad litum.

La aplicación de anestesia fue realizada por vía intramuscular aplicando $0,1 \mathrm{ml} / 200 \mathrm{~g}$ de peso del animal, utilizando partes iguales de Ketamina y Xilazina. Quince minutos después de aplicada la técnica anestésica se inyecto atropina $0,5 \mathrm{mg}$ en dosis de $0,08 \mathrm{mg} / \mathrm{kg}$, vía cutánea.

Procedimiento Quirúrgico. El procedimiento quirúrgico fue realizado de acuerdo al protocolo diseñado para la investigación el cual exigía que el día previo a la cirugía todos los animales fueran sometidos a tricotomía del sector a intervenir y pesaje para definir calculo farmacológico.

Al momento de la intervención quirúrgica, el área fue manejada con técnicas de asepsia y antisepsia con solución tópica de povidona iodada al $10 \%$. Se realizó una incisión de $4 \mathrm{~cm}$ en la región correspondiente al sector medial de la tibia (utilizándose ambas tibias del animal), en su tercio proximal, con hoja de bisturí No 15; se realizó divulsión de tejidos blandos y desperiostización hasta obtener total exposición ósea.

Posteriormente, se realizaron dos perforaciones de 2,6 mm de diámetro cada una, momento en que se aplico irrigación con suero fisiológico de forma profusa. Fueron generados dos defectos óseos en cada tibia, los cuales fueron organizados en cuatro grupos:

Grupo I: relleno con coágulo sanguíneo

Grupo II: relleno con coágulo sanguíneo cubierto por membrana de colágeno

Grupo III: relleno con sustituto óseo

Grupo IV: relleno con sustituto óseo cubierto por membrana de colágeno

Luego de la aplicación del modelo experimental se realizó sutura en planos a partir del periostio utilizando nylon 4.0, se aplico penicilina benzatina y se mantuvieron en sus ubicaciones iniciales con medidas de soporte y alimenta- ción habitual; la eutanasia de los animales fue realizada con sobredosis de Ketamina y Xilazina en el periodo de 21 y 42 días posteriores a la intervención quirúrgica.

Selección de Membrana y Substituto Óseo. Se utilizó membrana reabsorbible de colágeno (BioMend - Calcitek A company of Suizer Medical), fabricado a partir del tendón flexor bovino, de superficie plana y con aspecto texturizado, caracterizada como semi-oclusiva por presentar poros de 0,004 $\mathrm{mm}$. El substituto óseo utilizado en esta investigación fue sulfato de calcio hemi-hidratado de grano mediano y solución de dilución aceleradora (Capset, Lifecore Biomedicals, Inc., Chaska, MN, USA).

Análisis Histológico e Histomorfométrico. Después de la eutanasia, los defectos injertados fueron retirados con motor de baja rotación, respetando $5 \mathrm{~mm}$ de margen de seguridad desde el limite del defecto. Las piezas fueron fijadas en formol al $10 \%$ durante $24 \mathrm{~h}$, con temperatura ambiente; luego fueron sometidas a fase de descalcificación con citrato de sodio al $20 \%$ y ácido fórmico al $50 \%$ durante 52 días. Al finalizar el proceso, se realizaron cortes seriados de $6 \mathrm{~m}$, utilizando hematoxilina eosina para realizar las evaluaciones en microscopio óptico. Se realizó histología descriptiva para caracterizar la reparación óseo de cada defecto con magnificaciones de 10x y 50x.

Los datos recolectados fueron analizados mediante un test de varianza y test de Turkey considerando un nivel de significancia del $5 \%$.

\section{RESULTADO}

\section{Análisis Descriptivo}

\section{Periodo 21 Días}

Grupo I: La cavidad se encontraba rellena de tejido conectivo con un discreto proceso inflamatorio; Se observo formación ósea incipiente con mantención de los límites del defecto generado.

Grupo II: Los defectos presentan un comportamiento semejante al grupo I; el área mas superior del defecto presentaba importante cantidad de tejido conectivo, mientras que en el área interior se observaba presencia de tejido conectivo en conjunto con áreas de formación ósea.

Grupo III: Se identificaba claramente el limite entre el defecto óseo y el relleno utilizado; los márgenes presentan áreas de remodelación; en algunas muestras se observó la presen- 
cia de reacción inflamatoria y en otras se observó trabéculas óseas en neoformación rodeadas de tejido conectivo.

Grupo IV: Se identifica la diferencia entre el defecto y el relleno utilizado; se observó áreas de remodelación en el límite del defecto; en el interior del defecto se observó formación ósea en el área superior, próximo a la membrana.

\section{Periodo 42 Días}

Grupo I: Existen claras áreas de neoformación ósea, con aspecto organizado y con un área difusa en el limite del defecto generado lo cual puede asociar un nivel de integración ósea (Fig. 1).

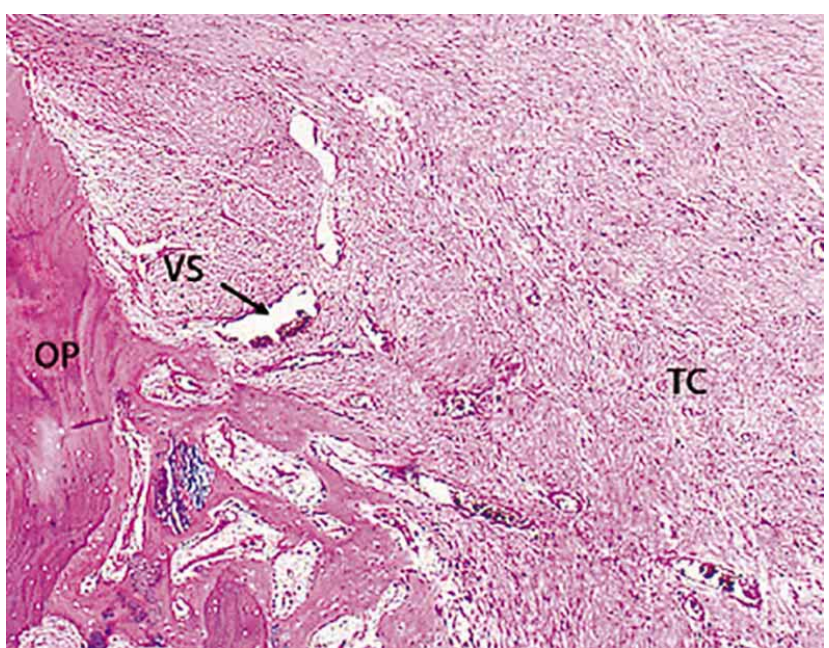

Fig. 1. Defecto creado en tibia de conejo estabilizado con coágulo sin membrana en el periodo de 42 días; se observa la presencia de tejido conectivo (TC), algunos vasos sanguíneos y la presencia de el hueso periférico (OP) al defecto creado.

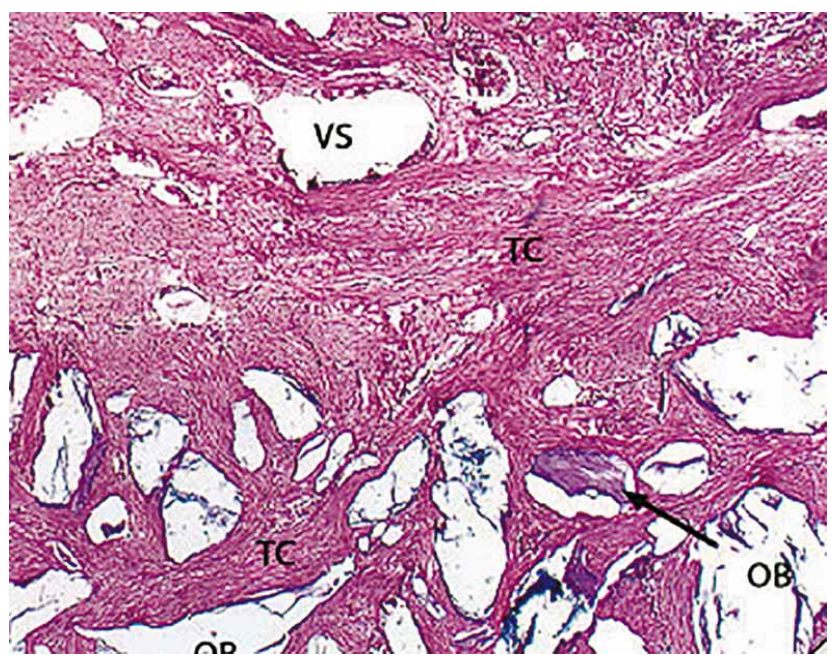

Fig. 2. Defecto creado en tibia de conejo tratado con relleno de sulfato de calcio cubierto con membrana en el periodo de 42 días; se observa la presencia de tejido conectivo, remanentes del biomaterial y formación de hueso rodeando la zona del biomaterial.
Grupo II: El tejido óseo neoformado existente y ocupa la mayor parte del defecto generado; la porción mas superior presenta aun indicios de presencia de tejido conectivo.

Grupo III: Cavidad rellenada con alta presencia de hueso neoformado, presencia de tejido conectivo; se observan trabéculas ósea que rodean tejidoconectivo y se observan restos existentes del material implantado.

Grupo IV: Se observe el límite entre la cavidad del defecto y el hueso nativo adyacente; el defecto se encuentra con amplias extensiones de tejido conectivo óseo, organizado y maduro, junto a la incorporación de tejido conectivo rodeado por tejido óseo neoformado (Fig. 2).

Análisis Histomorfométrico. Los resultados revelaron que hubo mayor formación ósea en la segunda etapa de mediciones. Para la primera etapa de 21 días, entre el grupo I y II no existió diferencias significativas, mientras que entre el grupo III y IV tampoco lo hubo. Al comparar los grupos de coágulo (I y II) con los del relleno (III y IV) existió diferencias significativas entre ellos $(\mathrm{p}=0,041)$. La Tabla I muestra los datos obtenidos en los análisis realizados para los diferentes periodos de eutanasia.

En el análisis del segundo periodo de 42 días, una situación semejante se presentó con ausencia de relación estadísticamente significativa entre el grupo I y II; el grupo IV fue superior a los otros $(\mathrm{p}=0,031)$ mostrando mayor cantidad de formación ósea; el grupo III fue superior a los grupos I y II ( $p=0,042)$, aunque fue significativamente menor a los resultados obtenidos en el grupo IV.

Tabla I. Distribución de los resultados de histomorfometría en base a análisis de puntos en las láminas histológicas seleccionadas para cada grupo.

\begin{tabular}{ccccc}
\hline \multirow{2}{*}{ Grupo } & \multicolumn{2}{c}{ 21 días } & \multicolumn{2}{c}{ 42 días } \\
\cline { 2 - 5 } & $\boldsymbol{X}$ & $\boldsymbol{D E}$ & $\boldsymbol{X}$ & $\boldsymbol{D E}$ \\
\hline I & 27,66 & 3,77 & 39,86 & 3,60 \\
II & 29,20 & 1,96 & 42,86 & 1,74 \\
III & 37,13 & 2,70 & 56,53 & 3,52 \\
IV & 37,86 & 2,58 & 64,13 & 2,92 \\
\hline
\end{tabular}

$\mathrm{X}=$ promedio; $\mathrm{DE}=$ Desviación Estándar.

\section{DISCUSIÓN}

El modelo de análisis empleado en esta investigación es el de regeneración ósea guiada, trabajando con defectos de diámetro de 2,6 mm en tibia de conejos. Este modelo ha sido empleado en otras investigaciones reportando resultados adecuados y reproducibles (Laventis et al.). 
La reparación ósea es influenciada por diferentes factores, tales como edad, estructura ósea, vascularización y tamaño del defecto (Dahlin et al., 1993). La condición estructural del injerto también ha sido vinculada a diferencias en la integración ósea, aunque la interface entre el injerto óseo y el lecho receptor no juega un rol importante cuando el injerto es de tipo autógeno (Netto et al., 2013a). Cuando materiales aloplásticos y heterógenos son utilizados, otros factores son protagonistas; Klüppel et al. (2013) demostraron que el tamaño de partículas del relleno óseo de matriz bovina tienen influencia importante en el modelo de reparación ósea, donde partículas de gran tamaño presentan una reparación ósea altamente retardada. Otro factor relevante puede ser el vehículo de instalación del material (Buchaim et al., 2012); en esta investigación fue utilizado el suero fisiológico como elemento de transporte, lo cual puede influenciar positivamente en la regeneración ósea ya que otros modelos de transporte pueden influenciar negativamente en la regeneración de tejidos (Netto et al., 2013b).

El uso de coágulo sanguíneo ha sido empleado en modelos experimentales como control y recientemente a sido aplicado en modelos clínicos para la regeneración ósea del seno maxilar, demostrando que cuando otros elementos biológicos confluyen de forma negativa (como una pobre calidad ósea y alto volumen del defecto), la exclusiva presencia de sangre sin injerto óseo, es insuficiente para llegar a la regeneración ósea (de Oliveira et al., 2013). Yang et al. confirmaron también esos análisis y realizaron un estudio de caso y control en sujetos con patología tumoral demostrando que el sulfato de calcio tendría un alto rendimiento en la recuperación de defectos óseos producidos por la resección y curetaje de los mismos. Kumar et al. (2013) realizaron un análisis sobre 15 pacientes tratando defectos óseos con relleno de sulfato de calcio describiendo complicaciones en algunos casos debido a infecciones en el sitio operatorio; sin embargo, las conclusiones establecen este material como seguro en su aplicación y función postoperatoria. Leventis et al., también concluyeron que el uso del sulfato de calcio colabora en la regeneración ósea de defectos en modelo animal.

En todos nuestros grupos evaluados, la neoformación ósea comienza desde las paredes laterales hacia la zona interior, al igual que lo observado por de Oliveira et al., confirmando que cuando el defecto óseo presenta una adecuada condición de irrigación sanguínea y con hueso cortical proporcionalmente menor, el tipo de material utilizado en el relleno puede ser menos importante que la calidad del tejido circundante (Choi et al.). El uso de la membrana biológica tiene como uno de sus objetivos el de complementar y auxiliar en el proceso de osteogénesis, contribuyendo como una barrera mecánica para la migración de células capaces de interferir en la formación ósea (Buser et al., 1995). Lopez et al. ya habían señalado que el uso de membranas seria útil en la regeneración, siendo mas eficiente que el no utilizarlas; de la misma forma en nuestros actuales resultados, se ha confirmado que la membrana biológica de colágeno podría influenciar positivamente en la regeneración ósea.

Otros estudios han apuntado a las complicaciones asociadas al uso de sulfato de calcio en defectos óseos. Friesenbichler et al. (2014) señalaron que sobre 40 pacientes tratados con este material existió complicaciones asociadas a procesos inflamatorios complejos y formación de quistes asociados al material; en nuestros resultados no se observó este tipo de complicaciones, pero es posible que en defectos mayores y procesos regenerativos complejos si existan situaciones de complicaciones asociadas. Estudios de imagenología podrían ser utilizado en los análisis de éxito y fracaso de procedimientos reconstructivos, sin embargo, Olate et al. señalaron que las radiografías no son útiles para estudiar avances, grados o cantidad de formación ósea cuando se utilizan materiales no autógenos en la reconstrucción, de forma que el estudio histológico es el mas conveniente en este ítem. Pacientes que son tratados con este material pueden tener limitaciones en el seguimiento adecuado de los resultados debido a estas limitaciones.

Bajo las condiciones de este estudio, se puede concluir que el sulfato de calcio aplicado en modelo animal es útil en la regeneración ósea; las membranas biológicas aumentan la posibilidad de regeneración ósea al compararse con coágulo sanguíneo.

DE OLIVEIRA, M.; OLATE, S.; POZZER, L.; VÁSQUEZ, B.; CANTIN, M. \& ALbERGARIA-BARBOSA, J. R. Bone repair using calcium sulfate in bone defects of rabbit tibiae. Int. J. Morphol., 32(4):1472-1476, 2014.

SUMMARY: Bone regeneration in the alveolar process and dental implant are widely used and there are a lot of different products. The aim of this research was to know the bone reparation associated to bone substitute with calcium sulfate and a biological membrane in created defects in rabbit tibiae. Were selected 12 rabbit between 3 and 6 month to be operated; using previous anesthesia protocols was do it a surgical defect in the right and left tibiae with $2.6 \mathrm{~mm}$ diameter; four groups were created: group I with blood clot fill, group II with blood clot fill plus biological membrane, group III with calcium sulfate and group IV with calcium sulfate plus biological membrane. The euthanasia was made in 21 and 42 days before surgery and was obtained histological plate using hematoxillin and eosin. The histomorphometry was made and statistical analysis using ANOVA and Turkey test with $\mathrm{p}<0.05$ to obtain statistical differences. Were observed in the all created defects a regular bone reparation; the group I and II, with blood clot, showed a minor bone reparation than group III and IV with calcium sulfate, but these last one show an important inflammatory 
process; the group IV presented the better results at 21 and 42 euthanasia days in term of bone formation. It's conclude that calcium sulfate can be used in bone reparation of minor defects and the biological membrane can be used in guide bone regeneration with success.

KEY WORDS: Biomaterial; Bone graft; Bone filling.

\section{REFERENCIAS BIBLIOGRÁFICAS}

Buchaim, R. L.; Andreo, J. C.; Rodrigues, A. D.; Buchaim, D. V.; Roque, D. D.; Roque, J. S. \& Rosa, G. M. Bovine Bone Matrix Action Associated With Morphogenetic Protein in Bone Defects in Rats Submitted to Alcoholism. Int. J. Morphol., 30(1):266-71, 2012.

Busser, D.; Ruskin, J.; Higginbottom, F.; Hardwick R.; Dahlin, C. \& Schenk, P. K. Osseointegration of titanium implants in bone regenerated in membrane-protected defects: a histologic study in the canine mandible. Int. J. Oral Maxillofac. Surg., 10(6):666-81, 1995

Choi, J. Y.; Jung, U. W.; Lee. I. S.; Kim, C. S.; Lee, Y. K. \& Choi, S. H. Resolution of surgically created three-wall intrabony defects in implants using three different biomaterials: an in vivo study. Clin. Oral Implant. Res., 22(3):343-8, 2011.

Dahlin, C.; Linde, A. \& Röckert, H. Simulation of early bone formation by the combination of an osteopromotive membrane technique and hyperbaric oxygen. Scand. J. Plast. Surg., 27(2):103-8, 1993 .

de Oliveira, G.; Olate S.; Cavalieri-Pereira, L.; Pozzer, L.; Asprino, L.; de Moraes, M. \& Albergaria-Barbosa, J.R. Maxillary sinus floor augmentation using blood without graft material. Preliminary results in 10 patients. J. Oral Maxillofac. Surg., 71:1670-5, 2013.

Friesenbichler, J.; Maurer-Erti, W.; Sadaghi, P.; Pirker-Fruehauf, U.; Bodo, K. \& Leithner, A. Adverse reactions of artificial bone graft substitutes: lessons learned from using tricalcium phosphate geneX. Clin. Orthop. Relat. Res., 472(3):976-82, 2014.

Klüppel, L.E.; Antonini, F.; Olate, S.; Nascimento, F. F.; Albergariabarbosa, J. R. \& Mazzonetto, R. Bone repair is influenced by different particle sizes of anorganic bovine bone matrix: a histologic and radiographic study in vivo. J. Craniofac. Surg., 24(4):1074-7, 2013.

Kumar, C. Y.; K. B. N.; Menon, J.; Patro, D. K. \& B. H. B. Calcium sulfate as bone graft substitute in the treatment of osseous bone defects, a prospective study. J. Clin. Diagn. Res., 7(12):29268, 2013.

Lopez, M. D.; Olate, S.; Lanata-Flores, A.; Pozzer, L.; Cavalieri-
Pereira, L.; Cantin, M.; Vásquez, B. \& de Albergaria-Barbosa, J. R. New bone formation in a bone defect associated to dental implant using absorbable or non-absorbable membrane in a dog model. Int. J. Clin. Exp. Pathol., 6(11): 2292-9, 2013.

Laventis, M. D.; Fairbaim, P.; Dontas, I.; Faratzis, G.; Valavanis, K. D.; Khaldi, L.; Kostakis, G. \& Eleftheriadis, E. Biological response to b-tricalcium phosphate/calcium sulfate synthetic graft material: an experimental study. Implant. Dent., 23(1):3743, 2014.

Netto, H. D.; Olate, S.; Klüppel, L. E.; do Carmo, A. M.; Vásquez, B. \& Albergaría-Barbosa, J. Histometric analyses of cancellous and cortical interface in autogenous bone grafting. Int. J. Clin. Exp. Pathol., 6(8):1532-7, 2013 a.

Netto, H. D.; Olate, S.; Klüppel, L.E.; Chaves, M. D. A. D.; Salgado, I. O.; Vásquez, B. \& Albergaria-Barbosa, J. R. Bone Regeneration in Critical Defect Treated with Bovine Inorganic Bone Matrix with two Different Carriers. Int. J. Morphol., 31(2):367-72, 2013b.

Olate, S.; Netto, H. D.; Kluppel, L. E.; Vásquez, B.; Chaves, M. D. A. M. \& del Sol, M. Comparison of bone graft healing between autogenous bone, blood clot and anorganic bovine bone matrix. Radiographic and histological analyses. Int. J. Morphol., 31(4):1257-62, 2013.

Yang, Y.; Niu, X.; Zhang, Q.; Hao, L.; Ding, Y. \& Xu, H. A comparative study of calcium sulfate artificial bone graft versus allograft in the reconstruction of bone defect after tumor curettage. Chin. Med. J. (Engl.), 127(17):3092-7, 2014.

Dirección para Correspondencia:

Prof. Dr. Sergio Olate

División de Cirugía Oral y Maxilofacial

Universidad de La Frontera

Claro Solar 115, Oficina 420

Temuco

CHILE

Email: sergio.olate@ufrontera.cl

Recibido : 16-08-2014

Aceptado: 05-10-2014 